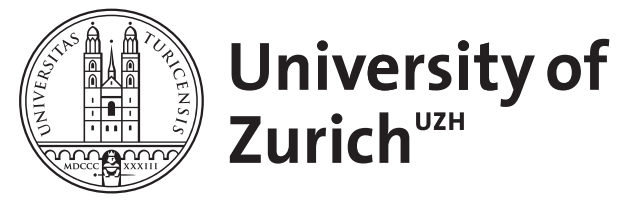

Zurich Open Repository and Archive

University of Zurich

University Library

Strickhofstrasse 39

CH-8057 Zurich

www.zora.uzh.ch

Year: 2008

\title{
Adipositastherapie bei Kindern und Jugendlichen in der Schweiz: Wo liegen
} die Hürden?

\author{
Hänggli, B ; Laimbacher, J ; Gutzwiller, Felix ; Fäh, David
}

Posted at the Zurich Open Repository and Archive, University of Zurich ZORA URL: https://www.zora.uzh.ch/4108

Journal Article

Originally published at:

Hänggli, B; Laimbacher, J; Gutzwiller, Felix; Fäh, David (2008). Adipositastherapie bei Kindern und Jugendlichen in der Schweiz: Wo liegen die Hürden? Schweizerische Ärztezeitung, 89(7):277-281. 


\section{Adipositastherapie bei Kindern und Jugend- lichen in der Schweiz: Wo liegen die Hürden?}

\author{
B. Hänggli, J. Laimbacher, \\ F. Gutzwiller, D. Fäh
}

\section{Zusammenfassung}

Im Rahmen einer medizinischen Dissertation in Zusammenarbeit mit dem Institut für Sozialund Präventivmedizin und dem Bundesamt für Gesundheit konnten 35 geeignete multidisziplinäre Adipositastherapiezentren für Kinder und Jugendliche identifiziert werden. Von den 30 Angefragten antworteten 27 (90\%). Ein Zentrum gab an, routinemässig stationär zu arbeiten, vier weitere arbeiten teilweise ambulant, die restlichen 22 nur ambulant.

In zwei Dritteln der Therapiezentren werden übergewichtige und adipöse Kinder, in einem Drittel nur adipöse Kinder behandelt. $85 \%$ machen medizinische Vorabklärungen, und 70\% formulieren weitere Aufnahmekriterien, wobei die meisten Zentren (11 von 27) eine Motivationsabklärung vornehmen. Das Durchschnittsalter der Patienten beträgt 12,9 Jahre (9,1-15,9). Das Geschlechterverhältnis ist ausgeglichen, auf vier Schweizer kommt ein Ausländer.

Die Mehrzahl der Zentren bietet eine ambulante Einzel- und Gruppentherapie an, die im Schnitt 10,7 Monate dauert. Die Therapieprogramme sind fast immer multidisziplinär, praktisch und theoretisch aufgebaut. Alle Zentren beziehen die Eltern in die Therapie mit ein, und fast alle führen eine Nachbetreuung durch. Durchschnittlich haben die Zentren 5,1 Jahre Erfahrung und betreuten im Schnitt 26,9 Patienten pro Jahr. Die meisten Zentren führen eine Evaluation durch, verwenden dafür aber stets eigene Kriterien.

Die durchschnittlichen Therapiekosten pro Kind (ambulant und stationär) werden mit Fr. 4844.- angegeben. Finanziert werden die Therapieprogramme in $85 \%$ durch die Eltern, mitfinanziert in $63 \%$ durch Fördergelder, in 59\% durch die Krankenkasse und in 26\% durch andere Finanzierungsmodelle (Mehrfachantworten möglich). Die Finanzierung wird in fast zwei Dritteln der Fälle als Grund angegeben, warum Kinder nicht in ein Programm gelangen. In 58\% der Fälle wird die Motivation des Kindes bzw. das familiäre Umfeld des Kindes angegeben und in 46\% das Argument, dass das Therapieangebot nicht bekannt ist. Gründe für das Scheitern einer Therapie werden in $84 \%$ der Fälle durch fami- liäre Probleme, in 80\% durch mangelnde Unterstützung der Eltern und in 52\% der Fälle durch mangelnde Motivation des Kindes erklärt (Mehrfachantworten möglich).

Die Arbeitsgruppe Adipositas der Schweizerischen Gesellschaft für Pädiatrie hat Grundlagen erarbeitet, die die Basis bildeten für den Antrag betreffend Festlegung der Leistungspflicht und Finanzierung der multiprofessionellen Therapieprogramme durch die Grundversicherung.

Im Herbst 2007 hat der Bundesrat entschieden, dass die Kosten der Behandlung von adipösen und übergewichtigen Kindern und Jugendlichen in multidisziplinären, ärztlich geleiteten Gruppenprogrammen von der obligatorischen Krankenpflegeversicherung übernommen werden müssen. In solchen Gruppenprogrammen können jedoch lediglich etwa 300-500 Patienten pro Jahr betreut werden. Die Finanzierung der dringend notwendigen Individualtherapie bleibt ungeklärt.

\section{Auch die Schweiz hat ein gewichtiges Problem}

Wie in vielen anderen europäischen Ländern steigt auch in der Schweiz die Prävalenz von Adipositas im Kindes- und Jugendalter: von 0,1\% pro Jahr in den 1980er Jahren auf 0,3\% pro Jahr in den späten 1990er Jahren [1]. 2002 lag die Prävalenz von Übergewicht bei Mädchen (Knaben) bei $19,1 \%(16,6 \%)$ und von Adipositas bei 3,7\% $(3,8 \%)$ [2]. Übergewichtige Kinder sind meist auch übergewichtige Erwachsene. Das Risiko von Übergewicht im Erwachsenenalter beträgt bei präpubertärem Übergewicht etwa 20-50\%, bei postpubertärem Übergewicht 50-70\%. In anderen Studien waren 70\% der adipösen Erwachsenen bereits als Kinder übergewichtig [3]. Somit stellen Gewichtsprobleme im Kindes- und Jugendalter auch erhebliche Risiken für damit verbundene chronische Erkrankungen dar, die meist viel später in Erscheinung treten.

\section{Übergewicht belastet nicht nur Sozialsysteme}

Durch Übergewicht und Adipositas entstehen direkte und indirekte Kosten. Diese wurden in der Schweiz im Jahr 2001 auf rund 2,7 Milliarden 
Franken geschätzt. Dabei fällt der grösste Teil $(98,4 \%)$ auf die 18 berücksichtigten Folgekrankheiten. Allen voran verursachen vor allem Diabetes Typ 2, koronare Herzerkrankung, Hypertonie und Depression die Kosten. Die durch Übergewicht und Adipositas verursachten Kosten sowie deren Anteil an den gesamten Gesundheitskosten (etwa 5\%) werden in den nächsten Jahren aufgrund der stetigen Zunahme von Übergewicht und Adipositas in der Schweizer Bevölkerung massiv zunehmen, falls keine entsprechenden Gegenmassnahmen eingeleitet werden [4]. Selbst die 2001 geschätzten Kosten von 2,7 Milliarden Franken liegen sehr wahrscheinlich weit unter den effektiven Kosten, weil sie auf Adipositasprävalenzen der Schweizerischen Gesundheitsbefragung (SGB) beruhen. In der SGB werden Körpergewicht und -grösse befragt und nicht gemessen, was zu einer Unterschätzung der effektiven Adipositasprävalenz in der Bevölkerung führt [5].

Das Winterthurer Institut für Gesundheitsökonomie der Zürcher Hochschule Winterthur [6] berechnete die direkten Kosten von Übergewicht und Adipositas auf zwischen 2 und 7\% der nationalen Gesundheitsausgaben. Für die Schweiz fehlen fundierte Daten, eine grobe Hochrechnung kommt auf direkte Kosten von 1077 bis 1615 Millionen Franken pro Jahr. Dazu kommen die zum Teil beträchtlichen indirekten Kosten durch Arbeitsausfall und vorzeitige Mortalität.

Übergewicht bedeutet jedoch nicht nur somatisches Risiko. Es gibt eine Reihe von Studien, die aufzeigen, wie insbesondere stark übergewichtige Personen im gesellschaftlichen, beruflichen und sozialen Leben diskriminiert werden [7]. Dies kann zu Minderwertigkeitsgefühlen und Essstörungen führen. Insbesondere Essattacken («binge eating») treten bei schwer übergewichtigen Personen gehäuft auf [8]. Die sozialen und psychischen Konsequenzen des Übergewichts können in einen Teufelskreis führen: Übergewicht verhindert oft sozialen Aufstieg, und niedrige soziale Stellung erhöht wiederum das Risiko für Übergewicht [9]. Kein Wunder, zeigt sich in der Schweiz wie auch in anderen westlichen Ländern eine inverse Beziehung der kindlichen Adipositas zum sozioökonomischen Status [1].

\section{Bisher keine einheitlichen Richtlinien für Therapieprogramme}

Angesichts der zunehmenden finanziellen und gesellschaftlichen Bedeutung von Übergewicht und Adipositas sah sich der Bund zum Handeln veranlasst. 2005 hat sich der Bundesrat zum Ziel gesetzt, die globale Strategie für Ernährung, Bewegung und Gesundheit der WHO für die Schweiz umzusetzen. Das BAG erarbeitet gegenwärtig in Zusammenarbeit mit verschiedenen Partnern ein an Schweizer Verhältnisse angepasstes nationales Programm «Ernährung und Bewegung». Der Schwerpunkt liegt bei Prävention und Gesundheitsförderung. Ein weiteres Ziel ist die Optimierung der Beratungs- und Therapieangebote zur Gewichtsreduktion. Ein bedarfsgerechtes und evidenzbasiertes Angebot an Beratung und Therapie soll den in der Schweiz lebenden Menschen und insbesondere den Kindern und Jugendlichen zur Verfügung stehen. Im Zentrum stehen Fragen der Wirksamkeit, Zweckmässigkeit und Wirtschaftlichkeit.

Deren Nachweis ist auch Voraussetzung für eine allfällige Aufnahme der Therapieangebote in die Leistungspflicht der Krankenversicherung. Die Ergebnisse einer randomisierten kontrollierten Studie, die die Wirksamkeit dieses Therapieansatzes dokumentieren, sind erst kürzlich publiziert worden [10]. Momentan sind solche Angebote bei Kindern nicht kassenpflichtig. Im Rahmen dieser Abklärungen wurde ein Stateof-Art-Bericht erstellt, der in Form einer medizinischen Dissertation in Zusammenarbeit mit dem Institut für Sozial- und Präventivmedizin Zürich publiziert wird. Das Ziel des hier auszugsweise veröffentlichten Berichts ist es, die Situation der verschiedenen multidisziplinären Therapieprogramme in der Schweiz zu erfassen. Die wichtigsten Resultate und Erkenntnisse dieser Publikation werden im Folgenden dargestellt.

\section{Bestehende Therapieprogramme in der Schweiz}

Fünfunddreissig multidisziplinäre Therapiezentren für übergewichtige und adipöse Kinder und Jugendliche konnten in der Schweiz identifiziert werden, wovon fünf Organisationen mit anderen Institutionen zusammenarbeiten. Von den 30 angefragten antworteten 27 (90\%): Fünf Zentren arbeiten stationär und ambulant, die restlichen 22 nur ambulant. Allerdings bietet nur ein Zentrum (Alpine Kinderklinik Davos) ein systematisches stationäres Programm an. Die restlichen vier Zentren bieten eine intensivierte, stationäre Phase inmitten eines ambulanten Programms an. Allerdings kommen diese stationären Phasen dort nur in Einzelfällen oder im Rahmen von Pilotprojekten zur Umsetzung.

\section{Behandelte Kinder und Jugendliche}

In zwei Dritteln der Therapiezentren werden übergewichtige und adipöse Kinder, in einem Drittel ausschliesslich adipöse Kinder behandelt. 
$85 \%$ (jeweils Mehrfachantworten möglich) der Zentren machen medizinische Vorabklärungen, und $70 \%$ formulieren weitere Aufnahmekriterien, wobei die meisten (11 von 27 Fällen) eine Motivationsabklärung genannt haben. Das Durchschnittsalter der Patienten beträgt 12,9 Jahre. Durchschnittlich sind sie mindestens 9,1 und maximal 15,9 Jahre alt. Das Geschlechterverhältnis ist ausgeglichen, auf vier Schweizer kommt ein Ausländer.

\section{Inhalt der Therapieprogramme}

Die Mehrzahl der Zentren bietet eine ambulante Einzel- und Gruppentherapie an, die im Schnitt 10,7 Monate (Bereich: 2-18 Monate) dauert. Die Therapieprogramme sind fast immer multidisziplinär (mit den Schwerpunkten Bewegung, Ernährung, Psychologie und Medizin), praktisch und theoretisch aufgebaut. Alle Zentren beziehen die Eltern in die Therapie mit ein, und fast alle führen eine Nachbetreuung durch. Die Erfahrung der einzelnen Zentren variierte stark: Durchschnittlich haben die Zentren 5,1 Jahre (0-27 Jahre) Erfahrung, betreuten bis anhin total 86,4 Patienten (7-400) bzw. 26,9 Patienten pro Jahr (4-290). Die meisten Zentren führen eine Evaluation durch, verwenden dafür aber stets eigene Kriterien.

\section{Kosten und Finanzierung}

Die durchschnittlichen Therapiekosten pro Kind werden mit Fr. 4844.- angegeben. Bei diesen Berechnungen wurden alle fünf Zentren mitberücksichtigt, die angegeben haben, Patienten auch stationär aufzunehmen. Mit Ausnahme der Alpinen Kinderklinik Davos wird dabei aber nur ein kleiner Therapieteil stationär vorgenommen, der Rest findet ambulant statt. Für die Alpine Kinderklinik Davos beliefen sich die durchschnittlichen Behandlungskosten auf Fr. 30240.-. Die 22 Zentren, die nur ambulant behandeln, gaben durchschnittliche Kosten von Fr. 1961.an. Diese Aufwendungen müssen durch die Eltern übernommen werden. Allenfalls zahlt der Krankenversicherer bei einem Spitalaufenthalt und je nach Versicherungsstand noch einen Teilbeitrag. Dies führt vor allem bei sozial schlechtergestellten Familien mit kleinem Haushaltseinkommen und ohne Zusatzversicherung zu Schwierigkeiten. In diesen Situationen müssen zur Kostendeckung Fördergelder oder andere Finanzierungsmodelle aushelfen.

Diese durch die Therapiezentren im Rahmen der Dissertation angegebenen Kosten sind tiefer als die von der Arbeitsgruppe Adipositas der Schweizerischen Gesellschaft für Pädiatrie kalkulierten Beträge. Dort wurde mit Kosten von
Fr. 4000.- bis 5000.- pro Jahr (inkl. Nachbetreuung und Evaluation, exkl. Eintritts- und Austrittsuntersuchung) für ein multiprofessionelles Therapieprogramm gerechnet [11]. Die tieferen Kosten haben verschiedene Gründe: Einerseits sind die Programme nicht standardisiert und deshalb auch sehr heterogen in ihrem Umfang und in der Nachbetreuung, was folglich zu sehr unterschiedlichen Kosten führt. Andererseits wurden die Kosten teilweise auch verzerrt dargestellt, da Quersubventionierungen stattfinden, die die durch die Eltern zu tragenden Kosten senken. Nicht zuletzt werden die Kosten wegen der bisher nicht einheitlich und systematisch durchgeführten Evaluationen falsch eingeschätzt.

\section{Finanzierung und familiäre Probleme als grösste Hürden}

Die Finanzierung wird in 65\% (jeweils Mehrfachantworten möglich) der Fälle als Grund angegeben, warum Kinder nicht in ein Programm gelangen. In $58 \%$ der Fälle wird die Motivation des Kindes bzw. das familiäre Umfeld angegeben und in $46 \%$, dass das Therapieangebot nicht bekannt ist. Gründe für das Scheitern einer Therapie werden in $84 \%$ der Fälle durch familiäre Probleme, in $80 \%$ durch mangelnde Unterstützung der Eltern und in 52\% der Fälle durch mangelnde Motivation des Kindes erklärt.

\section{Ausblick}

Adipositas bei Kindern und Jugendlichen ist in der Schweiz erst seit 2007 als behandlungsbedürftige und somit grundsätzlich als leistungspflichtige Krankheit anerkannt. Deshalb konnten erst jetzt verbindliche Richtlinien bezüglich Definition und Diagnostik sowie für die Therapie im Rahmen multiprofessioneller Programme erstellt werden. Die Arbeitsgruppe Adipositas der Schweizerischen Gesellschaft für Pädiatrie hat die entsprechenden Grundlagen erarbeitet. Diese bildeten die Basis für den Antrag an das Bundesamt für Gesundheit und an die Eidgenössische Leistungskommission (ELK) betreffend Festlegung der Leistungspflicht und Finanzierung der multiprofessionellen Therapieprogramme durch die Grundversicherung.

Am 22. Oktober 2007 hat der Bundesrat entschieden, dass die Kosten der Behandlung von adipösen und übergewichtigen Kindern und Jugendlichen in multidisziplinären, ärztlich geleiteten Gruppenprogrammen von der obligatorischen Krankenpflegeversicherung übernommen werden müssen. Per 1. Januar 2008 wurde die Krankenpflegeleistungsverordnung entsprechend geändert. In solchen Gruppenprogrammen können jedoch nur etwa 300-500 Patienten 


\section{Tabelle 1}

Identifizierte Therapiezentren - nach Regionen geordnet.

\begin{tabular}{|c|c|}
\hline Region/Ortschaft & Name des Therapieprogramms bzw. -zentrums (Homepage) \\
\hline \multicolumn{2}{|l|}{ Zürich } \\
\hline Winterthur & Adwin und Kinderklinik Winterthur (www.adwin.ch) \\
\hline Zürich & Club Minu (www.minuweb.ch) \\
\hline Zürich & Happy kids (www.bigawellfit.ch) \\
\hline Zürich & Knonaueramt knapp \\
\hline Zürich & Kinderspital Zürich (www.kispi.unizh.ch) \\
\hline Züricher Oberland & Minuzo (www.minuzo.ch) \\
\hline Zürich & $\begin{array}{l}\text { TRAP: Triemli-Adipositas-Programm (www.stadt-zuerich.ch/internet/ } \\
\text { triemli/home/klinken/kinder_jugendliche/adipositas.html) }\end{array}$ \\
\hline \multicolumn{2}{|l|}{ Nordwestschweiz } \\
\hline Aarau & OptiMahl (www.optimahl.ch) \\
\hline Riehen & AdiRi \\
\hline Basel & D-Club Castelmont \\
\hline Basel & $\begin{array}{l}\text { Universitätskinderspital beider Basel und TAKE: Training für adipöse Kinder } \\
\text { und ihre Eltern (www.ukbb.ch) }\end{array}$ \\
\hline Olten & Okapi (www.okapi-olten.ch) \\
\hline Solothurn & Abrakadabra \\
\hline Wohlen & Food 4 Teens (www.food 4 teens-wohlen.ch) \\
\hline \multicolumn{2}{|l|}{ Westschweiz } \\
\hline Fribourg & Fri move (www.prevention-fr.ch) \\
\hline Genf & Hôpitaux Universitaires de Genève (www.hcuge.ch) \\
\hline La Chaux-de-Fonds & éqkilo (www.sportsmile.ch oder www.fops.ch) \\
\hline Lausanne & Hôpital de l'enfance (www.hopital-enfance.ch) \\
\hline Porrentruy & Am Stram Grammes (www.jurasante.ch) \\
\hline Pully & Uscade (www.uscade.org) \\
\hline Vevey & Eurobesitas Ados (www.eurobesitas.ch) \\
\hline \multicolumn{2}{|l|}{ Ostschweiz } \\
\hline Münsterlingen & Kantonsspital Münsterlingen (www.stgag.ch) \\
\hline Schaffhausen & Shake (www.sh-ake.ch) \\
\hline St. Gallen & Adi Club und Kinderspital St. Gallen (www.kispisg.ch) \\
\hline \multicolumn{2}{|l|}{ Graubünden } \\
\hline Chur & Kinderklinik Kantonsspital Graubünden (www.ksgr.ch) \\
\hline Davos & Alpine Kinderklink Davos (www.kinderklinik-davos.ch) \\
\hline \multicolumn{2}{|l|}{ Zentralschweiz } \\
\hline Pfäffikon & Kjwj (www.kjwj.ch) \\
\hline Sarnen & Now or never \\
\hline \multicolumn{2}{|l|}{ Bern } \\
\hline Bern & WIM: Weniger is(s)t mehr (www.corinnespahr.ch) \\
\hline \multicolumn{2}{|l|}{ Tessin } \\
\hline Lugano & Ramo: ragazzi, alimentazione, movimento (www.mangiasano.ch) \\
\hline
\end{tabular}


pro Jahr in der Schweiz betreut werden, was bei über 47000 adipösen bzw. 247000 übergewichtigen Kindern und Jugendlichen in der Schweiz eine Minderheit darstellt.

Die Finanzierung der dringend notwendigen multiprofessionellen Individualtherapie ist hingegen immer noch ungeklärt. Diese Therapiemöglichkeit ist jedoch unabdingbar, da viele adipöse Kinder und Jugendliche für eine Gruppentherapie nicht in Frage kommen und in zentrumsfernen Regionen aufwendige Gruppenprogramme nicht angeboten werden können. Wenn Kinder und Jugendliche mit Adipositas von einer leistungspflichtigen multiprofessionellen Individualtherapie ausgeschlossen werden, so entspricht dies einer klaren Diskriminierung gegenüber Erwachsenen und ist ethisch nicht zu vertreten.

\section{Danksagung}

Wir danken dem Bundesamt für Gesundheit für die finanzielle Unterstützung im Rahmen des Berichtes sowie den Therapiezentren für ihre Beteiligung an der Studie. Ohne ihre Unterstützung wäre diese Arbeit nicht möglich gewesen.

\section{Referenzen}

1 Lasserre AM, Chiolero A, Paccaud F, Bovet P. Worldwide trends in childhood obesity. Swiss Med Wkly. 2007;137:157-8.

2 Zimmermann MB, Gubeli C, Puntener C, Molinari L. Overweight and obesity in 6-12 year old children in Switzerland. Swiss Med Wkly. 2004;134:523-8.
3 Suter PM. Ernährung, Lifestyle und Adipositas von Kindern und Jugendlichen in der Schweiz. In: Gesundheit Bf, ed. 5. Schweizerischer Ernährungsbericht. Bern, 2005:167-98.

4 Schneider H, Schmid A. Die Kosten der Adipositas in der Schweiz. Schlussbericht für das Bundesamt für Gesundheit (BAG). Bern; 2004.

5 Faeh D, Marques-Vidal P, Chiolero A, Bopp M. Obesity in Switzerland: do estimates depend on how body mass index has been assessed? Swiss Med Wkly. 2008, in press.

6 Federspiel B, Eisenring C, Kreuzer E. Bericht der Zürcher Hochschule Winterthur: Gesundheitsförderung Schweiz - Kernthema «Gesundes Körpergewicht» Ökonomische Perspektive; 2005.

7 Obesity: Preventing and managing the global epidemic. Genf; 2000 (reprinting 2004).

8 Schopper D. Gesundes Körpergewicht: Wie können wir der Übergewichtsepidemie entgegenwirken? Wissenschaftliche Grundlagen für die Erarbeitung einer Strategie für die Schweiz. Bern; 2005.

9 Lamprecht M, König C, Stamm H. Gesundheitsbezogene Chancengleichheit mit Blick auf "psychische Gesundheit - Stress» und «gesundes Körpergewicht». Grundlagendokument im Auftrag von Gesundheitsförderung Schweiz; 2005.

10 Savoye M, Shaw M, Dziura J, Tamborlane WV, Rose P, Guandalini C, Goldberg-Gell R, Burgert TS, Cali AM, Weiss R, Caprio S. Effects of a weight management program on body composition and metabolic parameters in overweight children: a randomized controlled trial. JAMA. 2007; 297:2697-704.

11 Sempach R, Farpour-Lambert NJ, l'Allemand D, Laimbacher J. Therapie des adipösen Kindes und Jugendlichen: Vorschläge für multiprofessionelle Therapieprogramme. Paediatrica. 2007;18:33-7. 